\title{
El impacto de la cultura nacional sobre la cultura organizacional*
}

\section{The Impact of National Culture on the Organizational Culture}

Recibido: febrero 14 de 2009 ｜ Revisado: mayo 20 de 2009 | Aceptado: mayo 30 de 2009

\author{
ALICIA OMAR ** \\ Consejo Nacional de Investigaciones Científicas \\ y Tecnológicas, Argentina \\ Alicia Florencia UrTeAgA *** \\ Universidad Nacional de Entre Ríos, Argentina
}

* El presente trabajo se realizó en el marco del proyecto PID-0506-CONICET.

** Instituto de Investigaciones-Facultad de Humanidades y Artes, Universidad Nacional de Rosario, Italia 1365 (2000) Rosario-Argentina. Correo electrónico: agomar@arnet.com.ar

**** Facultad de Ciencias Agrarias, Universidad Nacional de Entre Ríos, Ruta 11, km 9,5 (3101) ParanáArgentina.

Correo electrónico: furteaga@fca.uner.edu.ar

\section{RES UMEN}

Se analizaron las relaciones entre la cultura nacional y la cultura organizacional en 16 empresas argentinas. La muestra estuvo integrada por 429 empleados (292 varones y 184 mujeres), con una edad promedio de 35 años. Los sujetos completaron un cuestionario de datos sociodemográficos y las escalas de cultura nacional y de prácticas organizacionales en sus respectivos lugares de trabajo. Los resultados obtenidos indicaron que las prácticas implementadas por las empresas reflejan, en término medio, los valores de la cultura de origen de la organización. Análisis de regresión múltiple mostraron que las empresas nacionales argentinas se caracterizan por prácticas estrechamente vinculadas con el colectivismo, alta distancia al poder y alta evitación de la incertidumbre. En tanto que las empresas privadas se destacan por la implementación de prácticas asociadas con el individualismo, baja distancia al poder, baja evitación de la incertidumbre, bajo paternalismo y bajo fatalismo. Se discuten los resultados a la luz de las fortalezas y debilidades de la investigación.

Palabras clave autores

Cultura nacional, valores, cultura organizacional, prácticas.

Palabras clave descriptor

Cultura corporative, nacionalismo y cultura, valores culturales.

\section{A B S T R A C T}

The relationships between national culture and organizational culture were analysed in 16 Argentinean companies. Sample was integrated by 429 employees (292 male, and 184 female), mean age 35 years old. Subjects completed, in their own work places, a socio-demographic questionnaire, a scale of national culture, and a scale of organizational practices. Results indicated that organizations adopt practices that, on average, reflect the cultural values of their country of origin. Multiple regression analysis showed that Argentinean national companies are characterized by practices closely associated with collectivism, high power distance, and high uncertainty avoidance. In contrast, Argentinean privatized companies adopted practices most linked to individualism, low power distance, low uncertainty avoidance, low paternalism, and low fatalism. The results are discussed in light of its strengths and weaknesses, and a new agenda for future research is suggested. Key words authors

National Culture, Values, Organizational Culture, Practice.

Key words plus

Corporate Culture, Cultural Nationalism, Cultural Valúes. 
Desde la década de los años de 1990, la creciente globalización de la economía ha hecho que los procesos de fusión, expansión y adquisición de empresas se convirtieran en procedimientos cada vez más frecuentes, en el escenario mundial. Estos procesos basados, casi exclusivamente, en criterios económicos y competitivos han descuidado, por lo general, los aspectos humanos y culturales subyacentes. De allí que ha sido frecuente observar los problemas de adaptación, tanto de los empleados a prácticas organizacionales oriundas de otros contextos culturales como de los gerentes provenientes de otras culturas, a las prácticas locales. La génesis de tales problemas, desde la óptica de los científicos sociales, debería buscarse en las diferencias de valores entre la cultura de origen de la empresa y la cultura local. Frente a este escenario, el estudio de la cultura nacional, y sus implicancias para las empresas y sus miembros, viene capturando la atención de psicólogos sociales y organizacionales.

El constructo "cultura" ha sido definido de muy diversas formas a través de los años. Hofstede (1980) la consideró como el conjunto de programas mentales que controlan las respuestas de un individuo en un contexto dado; el Proyecto GLOBO la definió como la matriz de motivos, valores, creencias y significados compartidos por los miembros de un grupo y transmitidos a través de las generaciones (House, Hanges, Javidan, Dorfman \& Gupta, 2004); en tanto que Alas y Tuulik (2007) la describieron como el sistema de creencias, ideologías, lenguaje, historia y herencia étnica compartidos.

A pesar que no existe consenso internacional sobre la definición del término, existe acuerdo entre los especialistas (Hofstede, 2001; Triandis, 2000), de que el núcleo de la cultura está constituido por los valores. Los valores son objetivos deseables y trans-situacionales, que orientan conductas y sirven como principios que guían la vida de las personas (Fisher \& Lowell, 2003). De allí que el estudio de la cultura se viene realizando principalmente a través de un conjunto de valores derivados de análisis teóricos y estudios empíricos (Alas \& Tuulik, 2007; Omar \& Urteaga, 2008; van Rekom, van Riel \& Wierenga, 2006). En uno de los primeros estudios en esta dirección, realizado con empleados de 53 subsidiarias de una gran compañía multinacional (IBM), Hofstede (1980, 2001) identificó cuatro valores básicos que fueron rotulados como distancia al poder, individualismo-colectivismo, evitación de la incertidumbre y masculinidad-femineidad.

La distancia al poder es el grado en que los miembros menos poderosos de la sociedad esperan que el poder se distribuya desigualmente. En países con una gran distancia al poder (por ejemplo, China) las organizaciones son muy centralizadas, la relación entre jefes y subordinados está estrictamente reglamentada, las desigualdades y los privilegios son normales y las diferencias salariales son muy marcadas. En cambio, en países con poca distancia al poder (Austria, Dinamarca) las relaciones interpersonales son más democráticas, las personas se relacionan como iguales a pesar de las posiciones formales que ocupan, y los subordinados se sienten con el derecho de criticar las decisiones tomadas por aquellos con más poder.

La evitación de la incertidumbre fue definida como el grado en el que los miembros de una cultura perciben y reaccionan frente a situaciones o amenazas desconocidas. Esta condición refleja la necesidad de predictibilidad y de reglas, tanto escritas como tácitas. Hofstede ha señalado que en las culturas con alta evitación de la incertidumbre, las conductas basadas en reglas tienden a ser inconsistentes, poco claras y complicadas. En cambio, en las culturas donde hay poca, las reglas se establecen sólo cuando son necesarias. Ejemplo de países que puntúan alto en evitación de la incertidumbre son Rusia, Taiwán, Ecuador, Colombia, Brasil, Argentina y los países árabes, entre otros. House et al. (2004), han demostrado que la evitación de la incertidumbre no varía significativamente con la edad, ni con la antigüedad laboral, pero sí lo hace con el tipo de ocupación.

El individualismo-colectivismo se refiere a la naturaleza de las relaciones que un individuo mantiene con su grupo. Los miembros de culturas colectivistas se caracterizan por mantener fuertes vínculos entre sí y por el convencimiento de que la lealtad incondicional al grupo será correspondida con la 
protección necesaria. En cambio, los miembros de culturas individualistas mantienen lazos afectivos débiles y confían más en sí mismas y en su familia inmediata, que en el grupo de pertenencia. En el ordenamiento elaborado por Hofstede, se ubicaron entre los países colectivistas: China, Japón, Grecia, Jamaica, Rusia, España, Argentina, Brasil, México, Colombia; en tanto que Estados Unidos de Norteamérica, Francia, Alemania, Noruega, Dinamarca, se situaron entre los individualistas. En términos generales, se trata de una dimensión vinculada con el desarrollo (Schimmack, Oishi \& Diener, 2005), ya que los países desarrollados aparecen como más individualistas que los países en vías de desarrollo.

La masculinidad-femineidad se refiere a la valoración diferencial de los papeles femeninos y masculinos. La masculinidad es característica de sociedades con roles bien definidos para hombres y mujeres. La femineidad se refiere a comportamientos donde los roles sociales de ambos sexos se superponen considerablemente. Japón, Alemania y Estados Unidos aparecen como los países con mayor puntaje en masculinidad, en tanto que Rusia, Grecia, México, Argentina, Bélgica, Francia, China, se agrupan entre los países más orientados a la femineidad.

A estos cuatro valores básicos descritos por Hofstede, más recientemente Aycan y sus colaboradores agregaron dos nuevas orientaciones axiológicas, las que identificaron como paternalismo y fatalismo. El paternalismo fue definido como el grado en el que las autoridades ofrecen orientación, apoyo y protección a sus subordinados los que, en contrapartida, retribuyen con lealtad y respeto (Aycan, Kanungo \& Sinha, 1999). Mientras que el fatalismo (Aycan et al., 2000) se vincula con la creencia de no tener el control sobre el resultado de las propias acciones, lo que transforma en inocuos los esfuerzos por alcanzar determinadas metas o planes a largo plazo.

\section{Las prácticas organizacionales como núcleo de la cultura organizacional}

La introducción de la expresión "cultura organizacional" en la literatura especializada se debe a
Pettigrew (1979), quien la definió como "un sistema de significados pública y colectivamente aceptado por un grupo dado, en un cierto período de tiempo" (p. 574). De acuerdo con Schein (2004), la cultura organizacional debe ser concebida como "... un patrón de presuposiciones básicas compartidas, aprendidas por un grupo y enseñadas a los nuevos miembros como un modo correcto de percibir, pensar y sentir en relación a los problemas del grupo" (p. 12). Berson, Oreg y Dvir (2005) enfatizaron que la cultura organizacional constituye una de las características organizacionales fundamentales, puede ser influenciada por los valores de los líderes y fundadores, y se manifiesta a través de las prácticas organizacionales.

Hofstede, Neuijen, Ohayv y Sanders (1990) fueron pioneros en destacar que las prácticas organizacionales constituyen el lado visible de la cultura y se expresan a través de símbolos, héroes y rituales. En tanto que los valores se asocian a sentimientos inconscientes, que no pueden ser directamente observados, aunque pueden ser revelados a través de comportamientos alternativos. A partir de tales presupuestos, estos autores desarrollaron una escala destinada a explorar los valores y las prácticas que caracterizan a la cultura organizacional. El referido instrumento incluyó tres dimensiones subyacentes a los valores (necesidad de seguridad, centralidad del trabajo y necesidad de autoridad) y seis dimensiones subyacentes a las prácticas: procesos vs. resultados (opone una rígida división de tareas, a una que permite variaciones en función de los objetivos); empleado vs. tarea (contrasta la preocupación por el elemento humano, con la preocupación por las tareas a ser ejecutadas); parroquial vs. profesional (orientada hacia la derivación de la identidad del empleado a partir de la organización o de su tipo de trabajo); sistema abierto vs. cerrado (contrapone un sistema de estructuración interna transparente y fácilmente comunicable a los nuevos miembros, a un sistema de difícil aprehensión, que apenas los más antiguos consiguen dominar); sistema rígido vs. flexible (diferencia entre un sistema que ejerce rígido control formal sobre los empleados y un sistema que da libertad y autonomía a los mismos) y normativa 
vs. pragmática (orientada por sus propias reglas o por las necesidades de los consumidores).

$\mathrm{Al}$ aplicar su escala para comparar la cultura de 20 organizaciones holandesas y dinamarquesas, Hofstede y su equipo (1990) observaron que las empresas estudiadas presentaban diferencias significativas en la mayoría de las prácticas, aunque se diferenciaban poco en cuanto a los valores. Constataron, además, que las prácticas organizacionales estaban significativamente correlacionadas con una variedad de tareas, el tipo de estructura y los sistemas de control característicos de cada empresa. Frente a tales resultados, los autores concluyeron que las prácticas diarias constituyen la esencia de la cultura organizacional y que, por lo tanto, la cultura de las empresas debe ser explorada a través de las prácticas que la tipifican, reservándose los valores para la caracterización de la cultura nacional.

Movido por el creciente interés sobre las posibilidades de las prácticas para influir sobre el comportamiento organizacional, Verbeke (2000) realizó una adaptación de la escala de prácticas organizacionales de Hofstede et al. (1990), incorporando nuevos ítems y sometiéndola a un intenso programa de validación. Tales procedimientos dieron por resultado un instrumento con seis subescalas, de las cuales cuatro (procesos vs. resultados; empleado vs. tarea; sistema abierto vs. cerrado; sistema rígido vs. flexible) mantuvieron las denominaciones dadas por Hofstede, en tanto que las dos restantes fueron identificadas como responsabilidad social vs. auto-interés (similar a la dimensión normativa vs. pragmática de la escala original), y mercado vs. interna (referida a la implementación o no de prácticas orientadas hacia la competición y conquista de clientes). Estudios realizados con su nueva escala llevaron a Verbeke (2000) a concluir que las prácticas son relativamente independientes de los valores básicos de la organización, aunque sean moldeadas por esos valores. En línea con tal postura, en un estudio sobre las prácticas organizacionales de empresas tailandesas locales y de empresas tailandesas afiliadas a empresas norteamericanas, Chow, Harrison, McKinnon y Wu (2002) verificaron que estas últimas adoptaban prácticas orientadas hacia los resultados y hacia el mercado, en un grado significativamente mayor que las primeras. Tales resultados fueron interpretados como una demostración de la influencia de la cultura nacional en la cual la empresa se originaba sobre las prácticas adoptadas.

En lo que hace específicamente a las relaciones entre la cultura nacional y las prácticas organizacionales implementadas por las empresas, de la revisión de la bibliografía surgen posturas controversiales. Por un lado, se señala que las empresas desarrollan sus propias culturas y tienden a atraer empleados con similares características, incluyendo sus valores (Johns, 2006). De allí que muchas investigaciones están focalizadas sobre la congruencia entre los valores de los individuos y los de las organizaciones donde trabajan. Por otro lado, se sostiene que las organizaciones sufren influencias sistemáticas de su ambiente próximo y procuran adaptarse (Findler, Wind \& Mor Barak, 2007), por lo que serían afectadas por los valores de la cultura nacional en la que se insertan y no presentarían variaciones en cuanto a los valores que configuran su cultura interna. Tal continuidad valorativa entre la cultura nacional y la cultura organizacional ha sido defendida, entre otros, por Berson, Oreg y Dvir (2005), para quienes los valores de la sociedad se reflejan en la cultura interna del trabajo, y por Garibaldi de Hilal (2006), quien remarcó que los valores nacionales influyen sobre las prácticas desarrolladas dentro de las organizaciones.

A raíz de tales divergencias, la investigación orientada al esclarecimiento del interjuego entre los valores nacionales y las prácticas organizacionales no ha alcanzado resultados animadores. Circunstancia que amerita proseguir con esta línea de estudios que todavía demanda mayor evidencia empírica, sobre todo en países latinoamericanos, ya que los pocos resultados disponibles reflejan la situación de Estados Unidos o de algunos pocos países europeos. Y, básicamente, porque, durante la década de los años 1990, la mayoría de los países latinoamericanos se vieron inmersos en procesos de privatización de sus principales empresas estatales. Privatizaciones que, dejando de lado los aspectos financieros y legales que implicaron, se 
caracterizaron por la importación de gerentes desde los países inversores, los que llegaron trayendo sus valores, creencias y prácticas muchas veces distintos a los mantenidos por los grupos nacionales.

En consecuencia, el objetivo del presente estudio se orientó a explorar las posibles vinculaciones entre los valores y las prácticas que caracterizan la cultura organizacional de empresas nacionales y privatizadas argentinas. Con tal propósito se abordó la cultura nacional a través de los valores de distancia del poder, individualismo, evitación de la incertidumbre, paternalismo y fatalismo; en tanto que la cultura organizacional se evaluó a través de las siguientes prácticas: procesos vs. resultados; empleado vs. tarea; sistema abierto vs. cerrado; sistema flexible vs. rígido, y mercado vs. interna.

\section{Hipótesis}

En primer lugar, y partiendo de los hallazgos comunicados (Garibaldi de Hilal, 2006; House et al., 2004) se asumió que la cultura nacional de origen de una empresa afectaría su cultura organizacional, lo que se traduciría en la implementación de prácticas diferenciales, según se tratara de una empresa nacional o de una privada. Por lo tanto se postuló que:

Hipótesis 1: comparadas con las empresas privadas, las empresas nacionales argentinas implementarán con mayor frecuencia prácticas orientadas a los empleados y a los sistemas rígidos.

Hipótesis 2: comparadas con las empresas nacionales argentinas, las empresas privadas implementarán con mayor frecuencia prácticas orientadas a los resultados, al mercado y a los sistemas abiertos.

A su vez, a partir de las definiciones operacionales de las prácticas (Verbeke, 2000) y de cierta evidencia empírica disponible acerca de los valores nacionales (Alas \& Tuulik, 2007; Omar, 2006; Omar et al., 2007), se plantearon algunas potenciales relaciones entre ambos conjuntos de variables, las que se explicitan a continuación.
Las prácticas orientadas hacia los empleados denotan la inversión de la organización en sus recursos humanos, especialmente en lo que tiene que ver con su crecimiento y desarrollo profesional y a los intentos por establecer un involucramiento más personal entre gerentes y subordinados. En este sentido, sería esperable que en las organizaciones más colectivistas predominaran las prácticas orientadas hacia el empleado, por la importancia que el colectivismo le concede a la armonía grupal, al bienestar y al desarrollo de los miembros del grupo (Gilbreath \& Benson, 2004; Ramamoorthy, Gupta, Sardessai \& Flood, 2005). Asimismo, considerando el énfasis que el paternalismo pone sobre las relaciones de protección y cuidados entre jefes y subordinados (Aycan, 2004), sería esperable que en las organizaciones más paternalistas se adoptaran prácticas orientadas hacia el empleado. Por lo que se propuso que:

Hipótesis 3: cuanto mayor sea el paternalismo y el colectivismo, mayor será la adopción de prácticas orientadas al empleado.

Las prácticas que tipifican un sistema rígido se refieren a la adopción de un control excesivo sobre los empleados, especialmente en lo referente a la observancia de los horarios de trabajo y de las pausas realizadas durante el mismo. Por lo tanto, sería esperable que ellas presentaran una asociación positiva con la distancia al poder, en el sentido que tal control fuera utilizado como instrumento para mantener las distancias entre líderes y subordinados que caracteriza a tal dimensión (Alas \& Tuulik, 2007). Estas prácticas también podrían estar positivamente asociadas con la evitación de la incertidumbre, si se considera que la adopción de un control rígido constituiría una forma de impedir el surgimiento de situaciones inciertas y ambiguas (Grant, Christianson \& Price, 2007). Basándose en tales consideraciones se propuso la siguiente hipótesis:

Hipótesis 4: cuanto mayor la distancia al poder y la evitación de la incertidumbre, mayor será la adopción de prácticas rígidas. 
Las prácticas orientadas hacia los resultados implican permanente competencia con miras al logro de objetivos prefijados, lo que dificulta la colaboración y la ayuda mutua entre los empleados que ejercen diferentes funciones. Consecuentemente, sería de esperar que este tipo de prácticas se mostraran negativamente asociadas al colectivismo, por la importancia que éste le atribuye a la colaboración mutua y a la armonía intragrupal (Jackson, Colquitt, Wesson \& Zapata-Phelan, 2006). A su vez, deberían estar negativamente asociadas a la evitación de la incertidumbre, en virtud del énfasis que esta dimensión cultural pone sobre las situaciones estructuradas y sin ambigüedades (Alas \& Tuulik, 2007). Por lo tanto se hipotetizó que:

Hipótesis 5: cuanto menor el colectivismo y la evitación de la incertidumbre, mayor será la adopción de prácticas orientadas a los resultados.

Las prácticas orientadas hacia sistemas abiertos caracterizan organizaciones en las cuales gerentes y empleados discuten abiertamente errores y críticas, en lugar de hacerlo a escondidas. Por lo tanto, tales prácticas deberían presentar una correlación negativa con la distancia al poder, en la medida en que esta dimensión se define por la distancia que separa a líderes y subordinados, lo que probablemente dificulta una comunicación franca y abierta entre ellos. Asimismo, si se considera que las críticas pueden provocar conflictos en el interior de los grupos (Schimmack, Oishi \& Diener, 2005), el colectivismo podría estar negativamente asociado a tales prácticas ya que los gestores podrían adoptar prácticas orientadas hacia sistemas cerrados en un intento por evitar confrontaciones. En esta misma línea argumental, sería esperable que el paternalismo también presentara correlaciones negativas con las prácticas dirigidas a los sistemas abiertos, sobre todo por que los líderes paternalistas no se mostrarían dispuestos a las críticas, ya que al dispensar atención especial a los empleados, tales como cuidado y protección, esperarían su lealtad a cambio (Aycan, 2004). En consecuencia, se hipotetizó que:
Hipótesis 6: cuanto menor el colectivismo, el paternalismo y la distancia al poder, mayor será la adopción de prácticas orientadas a los sistemas abiertos.

Por último, las organizaciones que adoptan prácticas orientadas hacia el mercado tienen como meta prioritaria la atención de las necesidades y satisfacción del consumidor, razón por la cual se encuentran constantemente inmersas en un proceso competitivo que caracteriza la conquista de clientes. Por lo tanto, sería esperable que tales prácticas mostraran una asociación negativa con el colectivismo, ya que la valorización de los intereses grupales no fomenta la competición como un medio para el alcance de tales metas (Ramamoorthy, Gupta, Sardessai \& Flood, 2005). Asimismo, estas prácticas deberían asociarse negativamente con la evitación de la incertidumbre, en la medida en que la competición supone el involucramiento con situaciones nuevas y, a veces, desconocidas (Alas $\&$ Tuulik, 2007), y con el fatalismo, habida cuenta que el hecho de comprometerse con situaciones competitivas conlleva la creencia de cierta dosis de suerte, o sea, poco control sobre las situaciones (Aycan et al., 2000). Por lo que se supuso que:

Hipótesis 7: cuanto menor el colectivismo, la evitación de la incertidumbre y el fatalismo, mayor será la adopción de prácticas orientadas hacia el mercado.

\section{Método}

\section{Participantes y procedimientos}

Se recolectaron datos en 16 empresas argentinas -10 nacionales y 6 privadas-, ubicadas en la zona centro y sur del país. La muestra definitiva quedó integrada por 476 empleados, de los cuales el 53\% $(\mathrm{N}=252)$ pertenecía a empresas nacionales. El $42 \%$ eran mujeres; el promedio de edad de la muestra total fue de 35 años y el promedio de antigüedad laboral de 3,4 años. La mayoría de los sujetos tenían formación técnica o superior y el $9 \%$ de la muestra ocupaba cargos gerenciales. En cuanto a la actividad de las empresas, el 32\% eran fábricas 
o industrias, 27\% pertenecía al área de servicios (bancos, seguros, provisión de electricidad), 23\% al comercio y $18 \%$ a educación.

\section{Instrumentos}

Escala de cultura nacional (Omar, 2005). Se trata de una escala integrada por 20 ítems, con formato Lickert de 5 puntos, variando de "poco típico en mi país" a "muy típico en mi país". Explora las dimensiones axiológicas de individualismo (p. ej.: "la gente coopera con los demás miembros de la comunidad"), distancia del poder (p. ej.: "los jefes mantienen frecuentes contactos sociales con sus subordinados") y evitación de la incertidumbre (p. ej.: "las empresas informan claramente a sus empleados lo que esperan de ellos"), propuestas por Hofstede (2001) y las más recientes, paternalismo (p. ej.: "los jefes tratan a sus subordinados como si estuvieran tratando con sus propios hijos") y fatalismo (p. ej.: "la gente vive confiando en la suerte"), propuestas por Aycan y sus colaboradores (1999, 2000). En el presente estudio, los índices de consistencia interna ( $\alpha$ de Cronbach) de cada uno de estos factores variaron entre 0,823 y 0,955 (Tabla 1).

Escala de prácticas organizacionales (Omar, 2005). Se trata de una escala integrada por 25 ítems, con formato Lickert de 5 puntos, variando de "nada frecuente" a "muy frecuentemente". Explora tres de las prácticas de gerenciamiento de recursos humanos propuestas por Hofstede (1997), referidas a procesos vs. resultados (p. ej.: "existen mecanismos claros para medir los resultados obtenidos"), empleado vs. tarea (p. ej.: "los gerentes se preocupan por los problemas personales de los empleados), sistemas abiertos vs. cerrados (p. ej.: "cuando los empleados no están de acuerdo con alguna decisión, los gerentes escuchan sus opiniones"), y dos de las identificadas más recientemente por Verbeke (2000), referidas a sistemas flexibles vs. rígidos (p. ej.: "las tareas son desempeñadas de acuerdo con procedimientos previamente definidos") y mercado vs. empresa (p. ej.: "la empresa busca nuevos mercados para sus productos y servicios"). En el presente estudio, los índices de consistencia interna ( $\alpha$ de Cronbach) de cada uno de estos factores variaron entre 0,757 y 0,934 (Tabla 1).

\section{TABLA 1}

Dimensiones exploradas, número de ítems y coeficientes de consistencia interna ( $\alpha$ de Cronbach)

\begin{tabular}{llcc}
\hline & \multicolumn{1}{c}{ Dimensiones } & No ítems & $\alpha$ \\
\hline & $\begin{array}{l}\text { individualismo-colec- } \\
\text { tivismo }\end{array}$ & 4 & 0,955 \\
& $\begin{array}{l}\text { distancia jerárquica al } \\
\text { poder }\end{array}$ & 4 & 0,870 \\
$\begin{array}{l}\text { Cultura } \\
\text { Nacional } \\
\text { (20 ítems) }\end{array}$ & $\begin{array}{l}\text { evitación de la incerti- } \\
\text { dumbre }\end{array}$ & 4 & 0,823 \\
& paternalismo & 4 & 0,847 \\
& fatalismo & 4 & 0,920 \\
& procesos vs. resultados & 5 & 0,800 \\
& empleado vs. tarea & 5 & 0,854 \\
$\begin{array}{l}\text { Prácticas } \\
\text { Organiza- } \\
\text { cionales } \\
\text { (25 ítems) }\end{array}$ & $\begin{array}{l}\text { sistemas abiertos vs. } \\
\text { flexibles }\end{array}$ & 5 & 0,934 \\
& $\begin{array}{l}\text { mercado vs. interior } \\
\text { empresa }\end{array}$ & 5 & 0,757 \\
\hline
\end{tabular}

Fuente: elaboración propia.

\section{Procedimiento para la recolección de los datos}

La recolección de los datos se efectuó en el seno de las organizaciones que dieron su aval para participar en la investigación, y con los empleados que firmaron el protocolo de consentimiento informado. Los datos fueron reunidos en los lugares y horarios habituales de trabajo, de manera colectiva y/o individual, según los casos. Los participantes fueron informados de los objetivos de la investigación y se les garantizó el anonimato y la confidencialidad frente a la información que brindaran. Así mismo, recibieron las instrucciones sobre la mecánica de respuesta de los cuestionarios y fueron estimulados a ofrecer respuestas sinceras y a no dejar ninguna 
en blanco. Las eventuales dudas surgidas fueron aclaradas individualmente y en forma personalizada, durante la recolección de los datos. En todos los casos, junto a la nota de solicitud de autorización a las empresas participantes, se dejó expresa constancia del compromiso de devolución de los resultados, la que una vez finalizado el estudio adoptó la forma de una exposición pública en cada uno de los ámbitos empresariales.

\section{Análisis de los datos}

En primer lugar, se calcularon los índices descriptivos (medias y desviaciones típicas) correspondientes a las prácticas organizacionales, tanto para la muestra total, como separadamente para las empresas nacionales argentinas y las argentinas privatizadas. A partir de tales índices, y con el propósito de poner a prueba las hipótesis 1 y 2 , se procedió al cálculo de las correspondientes pruebas de diferencia de medias entre las prácticas características de cada tipo de empresa. Frente a los casos de diferencias significativas en la implementación de algún tipo de prácticas, y con el propósito de analizar las restantes hipótesis planteadas, seguidamente se procedió al cálculo de los correspondientes análisis de regresión múltiple para el grupo de empresas genuinamente nacionales o para el grupo de empresas privatizadas, según correspondiera. Para la ejecución de tales análisis de regresión, se ingresaron los valores culturales (individualismo, distancia al poder, evitación de la incertidumbre, paternalismo y fatalismo) como variables independientes, y las prácticas organizacionales como variables dependientes.

\section{Resultados}

El cálculo de las medias y de los desvíos típicos correspondientes a las prácticas implementadas por las empresas en estudio, posibilitaron la ejecución de las correspondientes pruebas $t$ de Student de diferencias de medias. Los resultados obtenidos dieron total apoyo a las hipótesis 1 y 2 desde el momento en que las empresas nacionales argenti- nas se diferenciaron significativamente de las privatizadas por su mayor orientación a las prácticas orientadas a los empleados $(t=6,54 ; \mathrm{p}<0.000)$ y a los sistemas rígidos $(t=3,47 ; \mathrm{p}<0,004)$. En tanto que las privatizadas mostraron un mayor empleo de prácticas orientadas a los resultados $(t=2,67 ; \mathrm{p}<$ $0,01)$; a los sistemas abiertos $(t=4,16 ; \mathrm{p}<0.002)$ $y$ al mercado $(t=3,50 ; \mathrm{p}<0.004)$.

Para poner a prueba las restantes hipótesis planteadas, que presuponían vinculaciones entre el tipo de práctica característico de una empresa y los valores prevalecientes entre sus miembros, se procedió al cómputo de un conjunto de análisis de regresiones múltiples, en los que los valores culturales básicos se ingresaron como predictores y cada una de las prácticas fue ingresada como variable dependiente (criterio). En el caso de las prácticas orientadas al empleado (Tabla 2) y a los sistemas rígidos (Tabla 3), sólo se trabajó con el grupo de las empresas nacionales argentinas, ya que ellas demostraron ser las que las adoptaban con mayor frecuencia. En el caso de las prácticas orientadas a los resultados (Tabla 4), a los sistemas abiertos (Tabla 5) y al mercado (Tabla 6), sólo se trabajó con el grupo de las empresas privatizadas, ya que ellas demostraron adoptar tales prácticas con mayor frecuencia.

\section{TABla 2}

Análisis de regresión múltiple. Variable dependiente: prácticas orientadas a los empleados

\begin{tabular}{lccccc}
\hline \multicolumn{1}{c}{$\begin{array}{c}\text { Variables } \\
\text { predictoras }\end{array}$} & \multicolumn{5}{c}{$\begin{array}{c}\text { Variable dependiente: } \\
\text { prácticas orientadas a los empleados }\end{array}$} \\
\hline & $\beta$ & $\mathrm{p}$ & & & \\
\hline Colectivismo & 0,332 & 0,001 & & & \\
$\begin{array}{l}\text { Distancia al } \\
\text { poder }\end{array}$ & 0,224 & 0,001 & $\mathrm{R}^{2}$ & $\Delta \mathrm{R}^{2}$ & $\mathrm{~F}(\mathrm{p})$ \\
$\begin{array}{l}\text { Evitación de } \\
\text { la incerti- } \\
\text { dumbre }\end{array}$ & 0,090 & n.s. & 0,124 & 0,113 & 3,$64 ; \mathrm{p}$ \\
Paternalismo & 0,306 & 0,001 & & & \\
Fatalismo & 0,071 & n.s. & & & \\
\hline
\end{tabular}

Fuente: elaboración propia. 
La hipótesis 3 postulaba que tanto el paternalismo como el colectivismo presentarían asociaciones significativas con las prácticas orientadas a los empleados. Los resultados (Tabla 2) mostraron que, efectivamente, el colectivismo $(\beta=0.332$, $p<$ 0.001) y el paternalismo $(\beta=0.306, p<0.001)$ se vinculan positivamente con esta práctica. Por lo tanto, la hipótesis 3 fue totalmente confirmada. Además, surgió una tercera dimensión (distancia al poder) positivamente asociada con las prácticas orientadas a los empleados $(\beta=.224, p<0.001)$, no prevista en las postulaciones iniciales.

TABLA 3

Análisis de regresión múltiple. Variable dependiente: prácticas orientadas a los sistemas rígidos

\begin{tabular}{|c|c|c|c|c|c|}
\hline \multirow[t]{2}{*}{$\begin{array}{c}\text { Variables } \\
\text { predictoras }\end{array}$} & \multicolumn{5}{|c|}{$\begin{array}{l}\text { Variable dependiente: prácticas } \\
\text { orientadas a los sistemas rígidos }\end{array}$} \\
\hline & $\beta$ & $\mathrm{p}$ & & & \\
\hline Colectivismo & 0,094 & n.s. & & & \\
\hline $\begin{array}{l}\text { Distancia al } \\
\text { poder }\end{array}$ & 0,218 & 0,003 & $\mathrm{R}^{2}$ & $\Delta \mathrm{R}^{2}$ & $\mathrm{~F}(\mathrm{p})$ \\
\hline $\begin{array}{l}\text { Evitación de } \\
\text { la incerti- } \\
\text { dumbre }\end{array}$ & 0,256 & 0,001 & 0,234 & 0,203 & $\begin{array}{l}7,575 ; \mathrm{p} \\
>0,000\end{array}$ \\
\hline Paternalismo & 0,240 & 0,001 & & & \\
\hline Fatalismo & 0,069 & n.s. & & & \\
\hline
\end{tabular}

Fuente: elaboración propia.

La hipótesis 4 postulaba que las prácticas orientadas a los sistemas rígidos serían adoptadas por organizaciones cuyos miembros estuvieran fuertemente orientados a la distancia al poder y a la evitación de la incertidumbre. Los resultados de la Tabla 3 indican que tales prácticas presentaron asociaciones en la dirección prevista con la distancia al poder $(\beta=0.218, p<0.003)$ y con la evitación de la incertidumbre $(\beta=0.256, p<0.001)$. Por lo que la hipótesis 4 también fue confirmada, aunque surgió una relación significativa, no prevista, con el paternalismo $(\beta=0.240, p<0.001)$.
TABLA 4

Análisis de regresión múltiple. Variable dependiente: prácticas orientadas a los resultados

\begin{tabular}{|c|c|c|c|c|c|}
\hline \multirow[t]{2}{*}{$\begin{array}{l}\text { Variables } \\
\text { predictoras }\end{array}$} & \multicolumn{5}{|c|}{$\begin{array}{c}\text { Variable dependiente: prácticas } \\
\text { orientadas a los resultados }\end{array}$} \\
\hline & $\beta$ & $\mathrm{p}$ & & & \\
\hline Colectivismo & $-0,416$ & 0,001 & & & \\
\hline $\begin{array}{l}\text { Distancia al } \\
\text { poder }\end{array}$ & 0,274 & 0,001 & $\mathrm{R}^{2}$ & $\Delta \mathrm{R}^{2}$ & $F(p)$ \\
\hline $\begin{array}{l}\text { Evitación de } \\
\text { la incerti- } \\
\text { dumbre }\end{array}$ & 0,038 & n.s. & 0,151 & 0,116 & $\begin{array}{c}4,40 ; p \\
>0,001\end{array}$ \\
\hline Paternalismo & 0,000 & n.s. & & & \\
\hline Fatalismo & 0,070 & n.s. & & & \\
\hline
\end{tabular}

Fuente: elaboración propia.

La hipótesis 5 predecía que la adopción de prácticas orientadas hacia los resultados se vincularía negativamente con el colectivismo y la evitación de la incertidumbre. Tal como lo muestra la Tabla 4, estas prácticas se asociaron negativamente con el colectivismo $(\beta=-0,416, p<0.001)$, sin embargo, y contrariamente a lo hipotetizado, no presentaron asociaciones significativas con la evitación de la incertidumbre $(\beta=0.038$, n.s. $)$. A su vez, surgió una asociación significativa, aunque no prevista, con la distancia al poder $(\beta=0.274, p<0.001)$. Por lo tanto, la hipótesis 5 fue parcialmente confirmada.

\section{TABLA 5}

Análisis de regresión múltiple. Variable dependiente: prácticas orientadas a los sistemas abiertos

\begin{tabular}{|c|c|c|c|c|c|}
\hline \multirow[t]{2}{*}{$\begin{array}{c}\text { Variables } \\
\text { predictoras }\end{array}$} & \multicolumn{5}{|c|}{$\begin{array}{l}\text { Variable dependiente: prácticas } \\
\text { orientadas a los sistemas abiertos }\end{array}$} \\
\hline & $\beta$ & $\mathrm{p}$ & & & \\
\hline Colectivismo & $-0,184$ & 0,003 & & & \\
\hline $\begin{array}{l}\text { Distancia al } \\
\text { poder }\end{array}$ & $-0,301$ & 0,001 & $\mathrm{R}^{2}$ & $\Delta \mathrm{R}^{2}$ & $\mathrm{~F}(\mathrm{p})$ \\
\hline $\begin{array}{l}\text { Evitación de } \\
\text { la incerti- } \\
\text { dumbre }\end{array}$ & 0,042 & n.s. & 0,187 & 0,155 & $\begin{array}{l}5,718 ; \mathrm{p} \\
>0,000\end{array}$ \\
\hline Paternalismo & 0,098 & n.s. & & & \\
\hline Fatalismo & 0,070 & n.s. & & & \\
\hline
\end{tabular}

Fuente: elaboración propia. 
La hipótesis 6 sostenía que las prácticas orientadas a los sistemas abiertos serían características de organizaciones con miembros escasamente orientados al colectivismo, al paternalismo y a la distancia al poder. Tal como lo muestra la Tabla 5 , las prácticas orientadas a sistemas abiertos, se relacionaron negativamente con el colectivismo $(\beta=-0.184, p<0.003)$ y con distancia al poder $(\beta=-0.301, p<0.001)$, pero, contrariamente a lo previsto, no presentaron ninguna relación con el paternalismo. Por lo tanto la hipótesis 6 fue parcialmente confirmada.

TABla 6

Análisis de regresión múltiple. Variable dependiente: prácticas orientadas al mercado

\begin{tabular}{|c|c|c|c|c|c|}
\hline \multirow[t]{2}{*}{$\begin{array}{l}\text { Variables } \\
\text { predictoras }\end{array}$} & \multicolumn{5}{|c|}{$\begin{array}{l}\text { Variable dependiente: } \\
\text { prácticas orientadas al mercado }\end{array}$} \\
\hline & $\beta$ & $\mathrm{p}$ & & & \\
\hline Colectivismo & $-0,191$ & 0,003 & & & \\
\hline $\begin{array}{l}\text { Distancia al } \\
\text { poder }\end{array}$ & 0,078 & n.s. & $\mathrm{R}^{2}$ & $\Delta \mathrm{R}^{2}$ & $F(p)$ \\
\hline $\begin{array}{l}\text { Evitación de } \\
\text { la incerti- } \\
\text { dumbre }\end{array}$ & $-0,343$ & 0,000 & 0,184 & 0,153 & $\begin{array}{l}5,674 ; p \\
>0,000\end{array}$ \\
\hline paternalismo & 0,032 & n.s. & & & \\
\hline fatalismo & $-0,436$ & 0,000 & & & \\
\hline
\end{tabular}

Fuente: elaboración propia.

La hipótesis 7 postulaba que la adopción de prácticas orientadas al mercado caracterizaría a las organizaciones cuyos miembros presentaran escasas tendencia al colectivismo, a la evitación de la incertidumbre y al fatalismo. Los resultados de la Tabla 6 indican que tal hipótesis fue totalmente confirmada desde el momento en que las prácticas orientadas al mercado presentaron asociaciones negativas con el colectivismo $(\beta=-0.191, p<$ $0.003)$, con la evitación de la incertidumbre $(\beta=$ $-0.343, p<0.000)$, y con el fatalismo $(\beta=-0.436$, $p<0.000)$.

\section{Discusión}

Los resultados obtenidos indican que la adopción de determinadas prácticas organizacionales por parte de las empresas radicadas en Argentina, se vincula estrechamente con el origen de la organización. En este sentido, las empresas nacionales argentinas se caracterizan por la implementación de prácticas orientadas al empleado y a los sistemas rígidos de gerenciamiento de sus recursos humanos. En cambio, las empresas privatizadas (mayoritariamente empresas de servicios, tales como aseguradoras, bancos, ferrocarriles, transportadoras mayoristas de gas o electricidad; que durante la década de los años de 1990 pasaron de la órbita del Estado nacional a manos de inversionistas extranjeros, que importaron sus gerentes y administradores), se caracterizan por la implementación de prácticas orientadas al mercado, a los resultados y a los sistemas abiertos. Tales hallazgos coinciden con las observaciones iniciales de Earley y Erez (1997) y las más recientes de House et al. (2004), quienes argumentaron que los gerentes implementan normas y prácticas dentro de las organizaciones que, en término medio, reflejan las normas y prácticas típicas de las organizaciones donde se formaron.

En sintonía con un importante cuerpo de estudios (Berson, Oreg \& Dvir, 2005; Garibaldi de Hilal, 2006), los resultados obtenidos indican que las prácticas implementadas por las empresas, reflejan los valores de la cultura de origen de la organización. En este sentido, se observó que las empresas genuinamente argentinas se caracterizan por prácticas estrechamente vinculadas con el colectivismo, distancia al poder y evitación de la incertidumbre, valores asociados a la Argentina (y a muchos otros países latinoamericanos) en el estudio transcultural realizado por Hofstede en 1980. Mientras que las empresas privatizadas se destacan por la implementación de prácticas organizacionales asociadas a un perfil axiológico caracterizado por el individualismo, baja distancia al poder, baja evitación de la incertidumbre, bajo paternalismo y bajo fatalismo, valores asociados a Estados Unidos de Norteamérica y a muchos países europeos, tanto por Hofstede (1980), como por 
Aycan et al. (2000). A continuación se efectúa un análisis más pormenorizado de las asociaciones observadas entre las prácticas organizacionales y los valores culturales.

Comenzando por las prácticas orientadas hacia los empleados, típicas de las empresas nacionales argentinas, se observa que éstas aparecen fuertemente vinculadas con el colectivismo y el paternalismo. Tales vinculaciones no hacen más que confirmar, por un lado, la primacía que el colectivismo le concede a la armonía intragrupal y al bienestar y satisfacción del empleado (Hattrup, Mueller \& Joens, 2007) y, por otro lado, enfatizan sobre las relaciones de protección y cuidados entre jefes y subordinados (Grant, Christianson \& Price, 2007), propias del paternalismo. No obstante, en el caso argentino, estas prácticas también aparecen asociadas con distancia al poder, lo que podría interpretarse como la aceptación, por parte de los empleados, de una desigual distribución de poder entre ellos y los jefes que deciden inversiones para mejorar su desenvolvimiento profesional. Vale decir que el perfil axiológico de los miembros de organizaciones que adoptan prácticas orientadas a los empleados, además de incluir rasgos del colectivismo y del paternalismo, incluirían rasgos vinculados con la distancia al poder.

Las prácticas que tipifican un sistema rígido, también características de las empresas nacionales argentinas, aparecen asociadas con alta distancia al poder y alta evitación de la incertidumbre. Vale decir que estas prácticas, basadas en rígidos controles del personal, serían utilizadas como instrumento de mantenimiento de la jerarquía entre líderes y subordinados (distancia al poder), así como una forma de impedir el surgimiento de situaciones inciertas y ambiguas (evitación de la incertidumbre). A su vez, y por la positiva correlación de estas prácticas con el paternalismo, pareciera que el mayor involucramiento emocional de los jefes con los empleados más que volverlos tolerantes frente a atrasos y otras infracciones de horario, los transformaría en controladores inflexibles.

Las prácticas orientadas a los resultados, típicas de las empresas privatizadas, son prácticas que demandan permanente competición para el logro de objetivos vinculados con patrones de cantidad o calidad. Del presente estudio, se observa que estas prácticas se asocian negativamente con el colectivismo (posiblemente porque el énfasis sobre los resultados y la autorealización es más característico del individualismo que del colectivismo), y positivamente con la distancia al poder. Aunque esta última relación no había sido hipotetizada, a la luz de los resultados obtenidos pareciera que el sostenimiento de tales prácticas requiere el reconocimiento de diferencias jerárquicas entre los jefes que definen los estándares de productividad y los empleados que deben alcanzarlos. Llamativamente, y a pesar de que se hipotetizó que entornos caracterizados por situaciones estructuradas y sin ambigüedades no serían propiciatorios para la adopción de estas prácticas, los resultados no mostraron asociaciones significativas con la evitación de la incertidumbre. Este hallazgo estaría indicando que, una vez implantadas, las prácticas orientadas a los resultados pueden sostenerse con independencia de las turbulencias organizacionales.

Las prácticas orientadas hacia sistemas abiertos, características de organizaciones en las que gerentes y empleados discuten abiertamente errores y críticas, se asocian negativamente con la distancia al poder (ya que esta dimensión probablemente dificulta una comunicación franca y abierta entre jefes y subordinados), y con el colectivismo (ya que las críticas pueden provocar conflictos en el interior de los grupos, por lo que en un intento por evitarlos, los jefes podrían adoptar prácticas orientadas hacia sistemas cerrados). Aunque también se esperaba que se asociaran negativamente con el paternalismo -ya que esta dimensión implica autoridad (Aycan, 2004) y el paternalista, como buen "dictador benevolente" (Northhouse, 1997) no está dispuesto a que discutan sus decisiones-, los datos reunidos no llegaron a mostrar ninguna asociación en esta dirección.

Las organizaciones que adoptan prácticas orientadas al mercado, en este caso las empresas privatizadas, tienen como meta prioritaria la atención de las necesidades y la satisfacción del consumidor, razón por la cual se encuentran constantemente 
inmersas en un proceso competitivo que caracteriza la conquista de clientes. Estas prácticas muestran asociaciones negativas con el colectivismo, ya que la competición privilegia los intereses individuales por encima de los grupales; con evitación de la incertidumbre, en la medida en que la competición supone frecuentemente el involucramiento con situaciones nuevas y, a veces, desconocidas; y con el fatalismo, en el sentido que el hecho de comprometerse con situaciones competitivas en general presupone la creencia de cierta dosis de suerte, o sea, poco control sobre los eventos.

\section{Aportes y limitaciones}

Como toda investigación empírica, el presente estudio presenta algunas fortalezas y limitaciones que merecen ser comentadas. Entre las limitaciones hay que señalar que la recolección de los datos estuvo basada en instrumentos autodescriptivos, por lo que los resultados podrían estar contaminados por la varianza del método común. Con miras a la superación de esta problemática, en futuras investigaciones se deberían incluir otras fuentes de exploración, tales como opiniones de los supervisores y clientes, así como observaciones sistemáticas, especialmente de las prácticas organizacionales; a pesar de que en investigaciones previas (Peters, Tjdens $\&$ Wetzels, 2004) se ha señalado que la evaluación de las prácticas organizacionales a partir de observaciones también podría verse afectada por algún componente de error. Otra de las limitaciones está relacionada con la exploración de los valores a través de métodos directos, vale decir, a través de mecanismos que sólo demandan evaluar la importancia de los mismos sobre una escala (Mumford, Connelly, Helton, Van Doorn \& Osburn, 2002). Si bien desde la perspectiva de Hofstede (2001), el enfoque directo es muy pertinente ya que supone que los sujetos no sólo son concientes de su orientación axiológica, sino que se comportan en función de los valores expresados, van Rekom, van Riel y Wierenga (2006) argumentan que el enfoque mixto puede proveer una mejor evaluación de los valores subyacentes al comportamiento individual. Por esto, en futuras investigaciones, debería considerarse la pertinencia de evaluar los valores a través de una combinación de medidas directas e indirectas. Una tercera limitación del estudio está referida a la posible contaminación de las respuestas por los efectos de la deseabilidad social. Sobre todo en grupos orientados al colectivismo, ya que los colectivistas suelen responder de manera socialmente deseable ( $\mathrm{Li}$, Triandis $\& \mathrm{Yu}$, 2006), tratando de complacer al entrevistador, aún cuando se les garantice el anonimato.

A pesar de las limitaciones expuestas, el presente estudio también exhibe algunas fortalezas. Los investigadores han enfatizado que una muestra que incluya una amplia gama de empleados provenientes de una variedad de organizaciones podría incrementar la validez externa. Consecuentemente, y con el propósito de aumentar la generalización de los resultados, se incluyó una amplia variedad de empleados representativos de un buen número de empresas y compañías. El uso de instrumentos de naturaleza émica, como los empleados aquí, desarrollados con base en las características idiosincrásicas de la población objetivo, posiblemente han permitido captar más apropiadamente el significado connotativo de los constructos investigados, y la intensidad y dirección de sus interrelaciones. Finalmente, y a pesar de la necesidad de refinamientos, la investigación realizada contribuye a conocer algo más acerca del impacto de la cultura nacional sobre la manifestación más visible de la cultura organizacional, como lo son las prácticas de manejo de sus recursos humanos.

\section{Referencias}

Alas, R. \& Tuulik, K. (2007). Cultural practices and values at the societal level in Estonia in comparison with neighbouring countries. Journal of Bussines and Management, 8, 39-44.

Aycan, Z. (2004). Key success factors for women in management in Turkey. Applied Psychology: An International Review, 53(3), 453-477.

Aycan, Z., Kanungo, R. N., Mendonca, M., Yu, K., Deller, J., Stahl, G. \& Kurshid, A. (2000). Impact 
of culture on human resource management practices: A 10-country comparison. Applied Psychology: An International Review, 49(1), 192-221.

Aycan, Z., Kanungo, R. N. \& Sinha, J. B. (1999). Organizational culture and human resource management practices: The model of culture fit. Journal of Cross-Cultural Psychology, 30, 501-526.

Berson, Y., Oreg, S. \& Dvir, T. (2005). Organizational culture as a mediator of CEO values and organizational performance. Academy of Management. Best Conference Paper BPS: FF1-6.

Chow, C. W., Harrison, G., McKinnon, J. L. \& Wu, A. (2002). The organizational culture of public accounting firms: Evidence from Taiwanese local and US affiliated firms. Accounting, Organizations and Society, 27, 347-360.

Earley, P. C. \& Erez, M. (1997). The transplanted executive: Why you need to understand how workers in other countries see the world differently. New York: Oxford University Press.

Findler, L., Wind, L. \& Mor Barak, M. (2007). The challenge of workforce management in a global society: Modeling the relationship between diversity, inclusion, organizational culture, and employee well-being, job satisfaction and organizational commitment. Administration in Social Work, 31(3), 63-94.

Fisher, C. \& Lovell, A. (2003). Business ethics and values. Boston: Prentice Hall.

Garibaldi de Hilal, A. V. (2006). Brazilian national culture, organizational culture and cultural agreement. Findings from a multinational company. International Journal of Cross Cultural, 6, 139-167.

Gilbreath, B. \& Benson, P. G. (2004). The contribution of supervisor behaviour to employee psychological well-being. Work and Stress, 18(3), 255-266.

Grant, A., Christianson, M. \& Price, R. (2007). Happiness, health, or relationships? Managerial practices and employee well-being tradeoffs. Academy of Management Perspectives, 32(3), 51-63.

Hattrup, K., Mueller, K. \& Joens, J. (2007). The effects of nations and organizations on work value importance: A cross-cultural investigation. Applied Psychology: An International Review, 56(3), 479-499.

Hofstede, G. (1980/2001). Culture's Consequences (1 ${ }^{\circ}$ and $2^{\circ}$ eds.). London: Sage Publications.
Hofstede, G. (1997). Cultures and Organizations. Software of the Mind. London: McGraw-Hill.

Hofstede, G., Neuijen, B., Ohayv, D. D. \& Sanders, G. (1990). Measuring organizational cultures: A qualitative and quantitative study across twenty cases. Administrative Science Quartely, 35, 286-316.

House, R. J., Hanges, P. J., Javidan, M., Dorfman, P. W. \& Gupta, V. (2004). Culture, leadership, and organizations. The Globe Study of 62 societies. London: Sage.

Jackson, C. L., Colquitt, J. A., Wesson, M. J. \& ZapataPhelan, C. P. (2006). Psychological collectivism: A measurement validation and linkage to group member performance. Journal of Applied Psycho$\log y$, 91, 884-899.

Johns, G. (2006). The essential impact of context on organizational behavior. Academy of Management Review, 31 (2), 386-408.

Li, S., Triandis, H. C. \& Yu, Y. (2006). Cultural orientation and corruption. Ethics and Behavior, 16, 199-215.

Mumford, M. D., Connelly, M. S., Helton, W. B., Van Doorn, J. R. \& Osburn, H. K. (2002). Alternative approaches for measuring values: Direct and indirect assessments in performance prediction. Journal of Vocational Behavior, 61, 348-373.

Northouse, P. G. (1997). Leadership: Theory and research. Tousand Oaks, CA: Sage.

Omar, A. (2005). La cultura organizacional de las empresas argentinas. Rosario: Conicet.

Omar, A. (2006). Justicia organizacional, individualismo-colectivismo y estrés laboral. Psicología y Salud, 16(2), 207-217.

Omar, A., Uribe, H., Ferreira, C., Assmar, E., Terrones, A. \& Galaz, M. (2007). Colectivismo, justicia y ciudadanía organizacional en empresas argentinas, mexicanas y brasileras. Revista Mexicana de Psicología, 24(1), 101-116.

Omar, A. \& Urteaga, F. (2008). Valores personales y compromiso organizacional. Enseñanza e Investigación en Psicología, 13(2), 353-372.

Peters, P., Tijdens, K. G. \& Wetzels, C. (2004). Employees' opportunities, preferences, and practices in telecommuting adoption. Information Management, 41(4), 469-482. 
Pettigrew, A. M. (1979). On studying organizacional cultures. Administrative Science Quartely, 24, 570. 581.

Ramamoorthy, N., Gupta, A., Sardessai, R. \& Flood, P. (2005). Individualism/collectivism and attitudes towards human resource systems: A comparative study of American, Irish and Indian MBA students. International Journal of Human Resource Management, 16, 852-869.

Schein, E. H. (2004). Organizational culture and leadership ( $8^{\circ}$ ed.). San Francisco: Jossey-Bass.

Schimmack, U., Oishi, S. \& Diener, E. (2005). Individualism: A valid and important dimension of cultural differences between nations. Personality and Social Psychology Review, 9, 17-31.

Triandis, H. C. (2000). Cross-cultural I/O Psychology at the end of the millennium. Applied Psychology: An International Review, 49(1), 222-226.

van Rekom, J., van Riel, C. B. M. \& Wierenga, B. (2006). A methodology for assessing organizational core values. Journal of Management Studies, 43, 175-201.

Verbeke, W. (2000). A revision of Hofstede et al.'s (1990) Organizational Practices Scale. Journal of Organizational Behavior, 21, 587-602. 